\title{
Fabrication, Characterization and Drug Release of Ciprofloxacin Loaded Porous Polyvinyl Alcohol/Bioactive Glass Scaffold for Controlled Drug Delivery
}

M Mabrouk ${ }^{1,2}$, AA Mostafa ${ }^{1,2 *}$, H Oudadesse ${ }^{1}$, AA Mahmoud ${ }^{3}$, AM Gaafar $^{4}$ and MI El-Gohary ${ }^{5}$

'University of Rennes 1, UMR CNRS 6226, 263 av. du général leclerc, 35042 Rennes Cedex, France

${ }^{2}$ Biomaterials Department, National Research Centre, Cairo, Egypt

${ }^{3}$ Pharmaceutical Technology National Research Centre (NRC), Cairo, Egypt

${ }^{4}$ Photo Chemistry Department, National Research Centre (NRC), Cairo, Egypt

${ }^{5}$ Physics Department, Faculty of Science, Al-Azhar University, Cairo, Egypt

\begin{abstract}
Composite scaffolds of polyvinyl alcohol (PVA) and/or quaternary bioactive glass (46S6 system) containing 5,10 and $20 \mathrm{wt} \%$ ciprofloxacin were prepared by lyophilisation technique. The porosity of the prepared scaffolds was measured by liquid displacement, $\mathrm{Hg}$-porosimeter and SEM. The structure and the nature of chemical bonds between atoms were examined by XRD and FTIR. They confirmed the incorporation of ciprofloxacin into the scaffolds. Biodegradation rate and drug release behaviour were conducted in phosphate buffer saline (PBS) at $\mathrm{pH}$ 7.4. A porous scaffold has been obtained with porosity up to $85 \%$. By increasing the glass contents and drug concentration in the prepared scaffold the porosity and the degradation rate decrease however, the compressive strength was enhanced. A sustained drug release pattern was observed from the optimized scaffold with a quasi-Fickian diffusion mechanism and it was able to deliver the drug in a prolonged release pattern which offers a distinguish treatment for osteomylitis as well as local antibacterial effect.
\end{abstract}

Keywords: Tissue engineering; Scaffolds; Ciprofloxacin; Drug release; Freeze drying; Polyvinyl alcohol

\section{Introduction}

Bioactive glass (BG) has various applications in repair and reconstruction of bone tissue; however, it has week mechanical properties especially in porous form. One approach to enhance the mechanical properties of materials is the elaboration of BG with polymer to form composites [1]. This way leads to an excellent combination between strength and toughness, as well as improved characteristics, when compared to their individual components [2]. The composites of BG/polymer are able to provide construct with excellent osteogensis and angiogenesis [1]. Application of a drug to a specific region using drug loaded scaffold produce high concentration of the drug in the required site of action which eliminate the side effects that prohibit the administration of large oral dose. Ciprofloxacin (Cip) is a fluroquinolone derivative, widely used as an antibiotic and in osteomyelitis because of its favorable penetration and bactericidal effect on all the probable osteomyelitis pathogens [3]. The main purpose of the current study was to develop and fabricate a construct of bioactive scaffold combining an antibiotic (ciprofloxacin) as a target drug delivery system. Characterization of these scaffolds before and after addition of the drug has been investigated by XRD, FTIR, mercury porosimeter, SEM, universal testing machine and UV-spectrophotometer.

\section{Materials and Methods}

\section{Synthesis}

The $46 \mathrm{~S} 6$ bioactive glass was prepared as previously reported [4] by using calcium silicate (Alfa Aesar), trisodium trimeta phosphate and sodium metasilicate pentahydrate (Sigma). The prepared glass was named MB. Polyvinyl alcohol (PVA) and polyvinyl alcohol/bioactive glass in molar ratio 1:2 PVA/MB scaffolds and scaffolds loaded with ciprofloxacin drug (PVA/MB-cip) were prepared by employing thermally induced phase separation technique (freeze drying). Firstly, $15 \%$ of PVA (Aldrich, M. wt $=67.000$ ) was dissolved in distilled water at $80^{\circ} \mathrm{C}$ for $2 \mathrm{hr}$. The prepared glass $\mathrm{MB}$ was added to polymer solution with continuous stirring at room temperature for $20 \mathrm{hr}$. Then, exactly 5,10 and $20 \%$ of ciprofloxacin drug were added to the batch of PVA and to that of PVA/MB composite with stirring for $1 \mathrm{hr}$. After that, the mixture was poured in moulds to form cylinders with diameters of 15 $\mathrm{mm} \times 10 \mathrm{~mm}$ and kept at $-18^{\circ} \mathrm{C}$ overnight, then transferred to a freeze dryer type Christ, Alpha 1-2 LD plus for $24 \mathrm{hr}$.

\section{Characterizations}

The porosity and the micro architecture of the prepared scaffolds were assessed by means of Mercury Intrusion Porosimetry (MIP) by using Poresizer 9320 V2.08, liquid displacement method and scanning electron microscopy (SEM) (Jeol JSM 6301).The phases and structure of each components of the prepared scaffold had been examined by XRD (Philips X-PERT with Cu Ka wave length of $1.5418 \AA$ ) and FTIR (Nicolet Magna-IR 550). Compressive strength was measured by universal testing machine type Lloyd. The release of the drug was constructed in PBS at pH 7.4 and drug concentration was followed using UV-spectrophotometer (Jenway 6705) at $277 \mathrm{~nm}$.

\section{Results and Discussion}

\section{Morphological and microstructural properties}

The morphology of the prepared scaffolds is presented in (Figure

*Corresponding author: AA Mostafa, University of Rennes 1, UMR CNRS 6226, 263 av. du général leclerc, 35042 Rennes Cedex, France, E-mail: amany.mostafa@univ-rennes1.fr

Received May 13, 2013; Accepted May 30, 2013; Published September 10, 2013

Citation: Mabrouk M, Mostafa AA, Oudadesse H, Mahmoud AA, Gaafar AM, et al (2013) Fabrication, Characterization and Drug Release of Ciprofloxacin Loaded Porous Polyvinyl Alcohol/Bioactive Glass Scaffold for Controlled Drug Delivery. Bioceram Dev Appl S1: 009. doi: 10.4172/2090-5025.S1-009

Copyright: $\odot 2013$ Mabrouk M, et al. This is an open-access article distributed under the terms of the Creative Commons Attribution License, which permits unrestricted use, distribution, and reproduction in any medium, provided the original author and source are credited. 
Citation: Mabrouk M, Mostafa AA, Oudadesse H, Mahmoud AA, Gaafar AM, et al. (2013) Fabrication, Characterization and Drug Release of Ciprofloxacin Loaded Porous Polyvinyl Alcohol/Bioactive Glass Scaffold for Controlled Drug Delivery. Bioceram Dev Appl S1: 009. doi: 10.4172/2090-5025.S1-009

Page 2 of 4

1). It could be noticed that all the prepared scaffolds have wide range of interconnected pores including macro, micro and nanopores as it is also confirmed by mercury porosimeter (Table 1). Scaffolds of PVA (Figure 1a) show highly interconnected pores with smooth pore walls. As the glass content added to the scaffold the porosity decreases and the pore walls becomes thicker (Figure 1b). Among several processing techniques, the freeze drying method was chosen since it could provide easy control of the pore structure [5]. The coexistence of macropores and microspores is not only favorable for the ingrowth of cells and new tissue but also beneficial to the exchange of nutrients and metabolic waste [6]. Addition of the drug with PVA scaffold is highly endows fiber formation and oriented the structure of the scaffold as shown in Figure 1c. However, the glass particles are clearly presented in Figure 1d.

Table 1 presents the results of porosity by MIP and liquid displacement method for PVA and PVA/MB scaffolds before and after drug loading. No significant difference between the two measuring techniques. It is also noted that the incorporation of ciprofloxacin affects the porosity percentage as it is decreased in PVA scaffold and increase in PVA/MB composite scaffold. This confirms that the added glass is affecting the average pore diameter and the array of the internal microstructure [7]. The prepared scaffolds showed well interconnected macropores with porosity in average of $73 \%$ and a pore size of more than $140 \mu \mathrm{m}$ that are suitable for bone substitute.

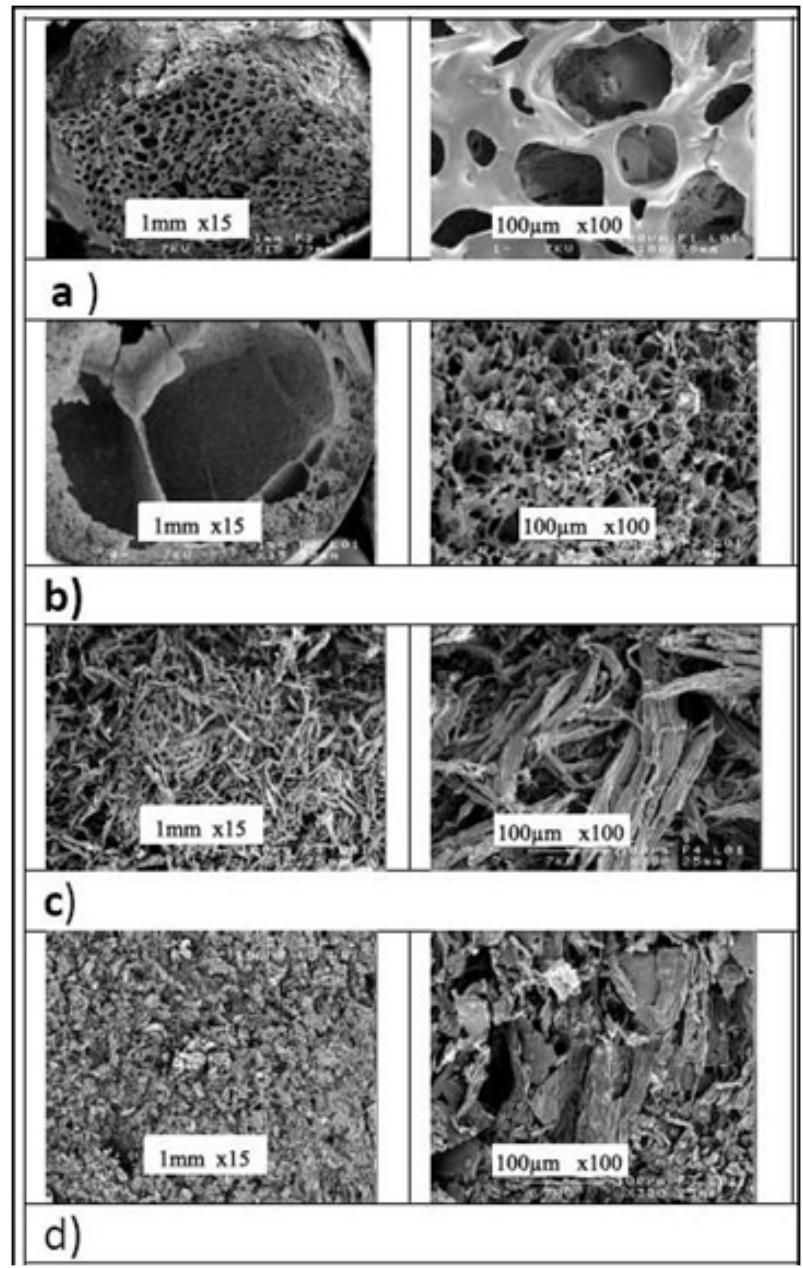

Figure 1: SEM images for a) PVA scaffold, b) 1: 2 PVA/MB scaffolds, c) PVA loaded with $20 \%$ of drug and d) PVA/MB loaded with $20 \%$ of drug with different magnifications.

\section{XRD before and after drug loading}

X-ray diffraction of PVA, MB, cip and 1:2 PVA/MB scaffolds with and without the drug are presented in (Figure 2). It could be noticed the amorphous structure of PVA, MB, the scaffold PVA/MB while, the crystalline structure of the cip drug is clearly noticed. When ciprofloxacin was entrapped into the scaffold matrix, its sharp crystalline peaks were overlapped with the halo of the surrounded polymer and the glass. The sharpness completely disappeared indicating that ciprofloxacin was successfully entrapped into the scaffold matrix system and forming a newly solid phase of scaffold/ ciprofloxacin with low crystallinity [8].

\section{FTIR before and after drug loading}

Figure 3 shows the FTIR of each constituent of the scaffold; PVA, $\mathrm{MB}$ and their composite in addition to cip drug, PVA loaded $20 \%$ cip and finally the composite scaffold PVA/MB loaded $20 \%$ cip, respectively. The IR spectrum of PVA proves the presence of "OH" group peak of

\begin{tabular}{|c|c|c|c|c|c|c|c|}
\hline \multirow{4}{*}{$\begin{array}{l}\text { Sample } \\
\text { Name }\end{array}$} & \multicolumn{2}{|c|}{$\begin{array}{c}\text { Pore Diameter } \\
\text { range (4V/A) }\end{array}$} & \multicolumn{5}{|c|}{ Porosity (\%) } \\
\hline & \multirow{3}{*}{$\mu \mathrm{m}$} & \multirow{3}{*}{$\mathrm{nm}$} & \multirow{3}{*}{ MIP } & \multirow{3}{*}{$\begin{array}{l}\text { Without } \\
\text { Drug }\end{array}$} & \multirow{2}{*}{\multicolumn{3}{|c|}{$\begin{array}{c}\text { Liquid Displacement } \\
\text { With Drug }\end{array}$}} \\
\hline & & & & & & & \\
\hline & & & & & $5 \%$ & $10 \%$ & $20 \%$ \\
\hline PVA & 139 & 6.2 & 88.14 & 85.47 & 72 & 69 & 66 \\
\hline 1:2 PVA/MB & 145 & 6.3 & 46.18 & 41.31 & 72 & 72 & 72 \\
\hline
\end{tabular}

Table 1: Porosity percentage and pore diameter measured by $\mathrm{Hg}$ - porosimeter and liquid displacement techniques.

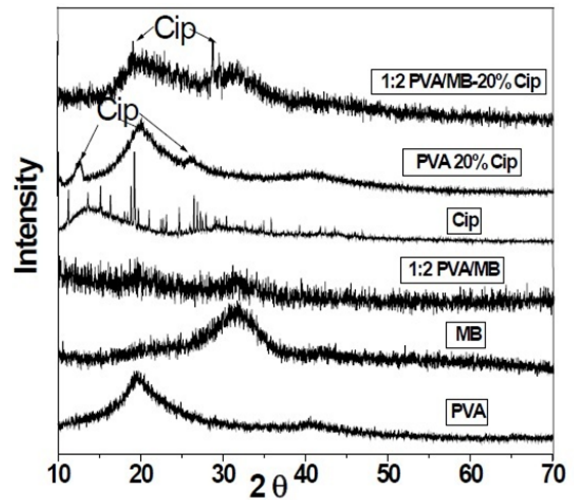

Figure 2: XRD of PVA, MB, cip and scaffold of PVA and 1:2 PVA/MB with and without $20 \%$ of ciprofloxacin.

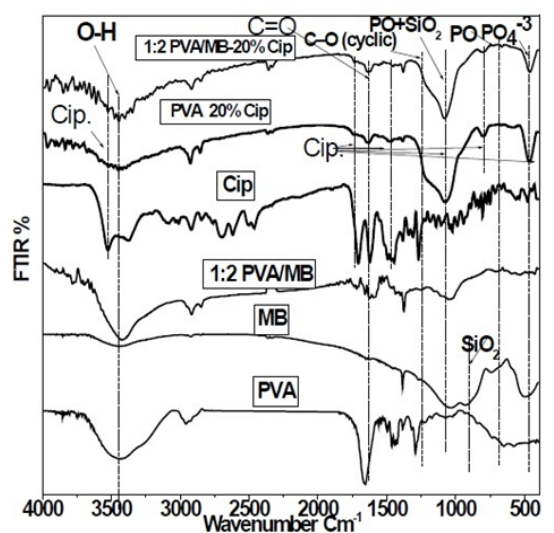

Figure 3: FTIR of PVA, MB, cip and scaffold of PVA and 1:2 PVA/MB with and without $20 \%$ of ciprofloxacin. 
Citation: Mabrouk M, Mostafa AA, Oudadesse H, Mahmoud AA, Gaafar AM, et al. (2013) Fabrication, Characterization and Drug Release of Ciprofloxacin Loaded Porous Polyvinyl Alcohol/Bioactive Glass Scaffold for Controlled Drug Delivery. Bioceram Dev Appl S1: 009. doi: 10.4172/2090-5025.S1-009

Page 3 of 4

alcohol between 3500 and $3450 \mathrm{~cm}^{-1}$, which was assigned to stretching vibration of $\mathrm{OH}$ group. In addition to some acidic groups as it is clear from the band at 1750 to $1700 \mathrm{~cm}^{-1}$ corresponding to $\mathrm{C}=\mathrm{O}$ stretching. In the drug cip spectrum, the "OH" group and carboxylic acid group are presented beside a band between 1650 and $1600 \mathrm{~cm}^{-1}$ assigned to quinolones in addition to the N-H band. A strong absorption peak between 1050 and $1000 \mathrm{~cm}^{-1}$ was assigned to C-F group. Elaboration of the drug with the PVA, cross linking occurs and the following facts are proved. In polymer scaffold loaded $20 \%$ cip drug the $\mathrm{OH}$ band decreases if compared by either the polymer or the drug that indicated of condensation reaction and elimination of $\mathrm{OH}$ group. However, the "CO" band in both the polymer and the drug almost vanished and replaced by newly formed band $\mathrm{C}-\mathrm{N}$ at around $1000 \mathrm{~cm}^{-1}$ which explains why the cross linked polymer/drug almost has no acidity if compared with both polymer and drug alone. A shorter band appeared in the region of $1500-1200 \mathrm{~cm}^{-1}$ that could be ascribed to the hydrated bonds with ciprofloxacin molecules [8]. The condensation of the drug with the PVA is explained by the mechanism demonstrated in scheme 1. It presents that the PVA could react with the drug (cip) in multiposition, to form the cross linking bridge. The active centers of PVA from 1-4 and for the drug sites from A, B and C, could be linked by the condensation reaction mechanism.

\section{Mechanical properties}

The mechanical behavior of the prepared scaffolds was characterized by determining the compressive strength before and after drug incorporation. The PVA scaffold alone even containing different amounts of the drug loading 5, 10 and 20\% exhibit low compressive strength as shown in (Figure 4). It could be noted that the compressive modulus increased by the addition of the glass with $0 \mathrm{wt} \%$ of the drug. However, a marked change could be observed, as the drug concentration increased from 5 to 10 and $20 \%$ even with no change in the porosity $\%$ as

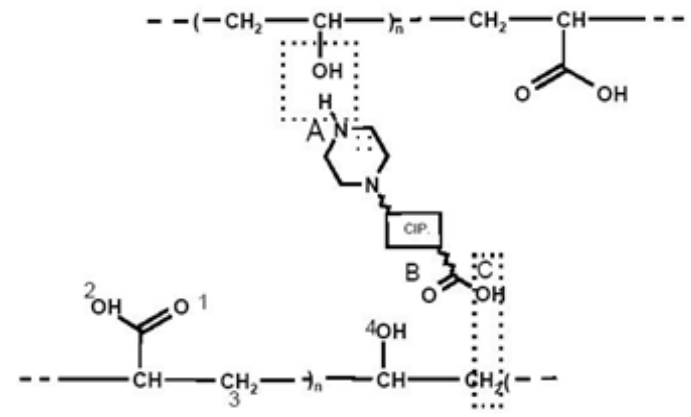

Scheme 1: Reaction mechanism between PVA and ciprofloxacin

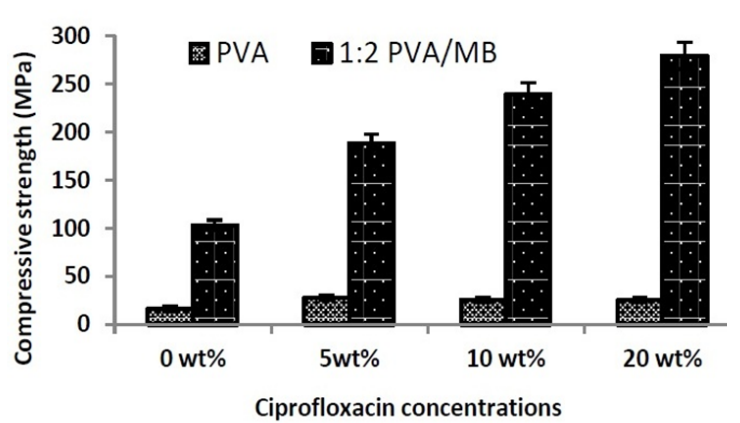

Figure 4: Compressive strength of the scaffolds before and after drug loading with different wt\%. previously mentioned. Flautre et al. [9] compared the interconnecting pore sizes of $30,60,100$ and $130 \mu \mathrm{m}$ and found that osteoconduction was greatest with $130 \mu \mathrm{m}$. Although greater pore size or interconnecting pore size leads to greater osteoconduction, the compressive strength of porous construct and clinical usefulness are reduced at larger pore sizes. This is not the case in this study as it is proved that addition of the drug added an advantageous of the mechanical properties at the same time with preserving the porosity through the induced cross linking without affecting of the drug efficiency.

\section{Degradation rate}

Biodegradation rate of the prepared scaffolds with and without drug was investigated in PBS at $\mathrm{pH} 7.4$ for different time intervals as shown in (Figure 5). Scaffold of PVA exhibit high degradation rate (100\% after 2 days). However, scaffold containing glass or that medicated, PVA/ MB and PVA $20 \%$ cip or PVA/MB-cip show delayed degradation. A relatively low degradation rate is much favorable for cell attachment and differentiation.

Furthermore, addition of the glass in the scaffold decreases the degradation rate due to the fact that incorporation of inorganic filler into polymer matrix decreases the porosity as confirmed by mercury porosimeter and liquid displacement methods and as documented before [10]. Drug loaded scaffold (PVA-cip) relatively decreases the biodegradation rate of the prepared scaffold with glass PVA/MB due to the great bounding ability of ciprofloxacin to PVA matrix as confirmed by XRD and FTIR [11]. The prepared scaffold could withstand up to two months before complete degradation, data not presented.

\section{Release behavior of ciprofloxacin}

Figure 6 shows the in-vitro release profiles of ciprofloxacin with different amounts 5, 10 and 20\% from the investigated scaffolds. The release behavior for ciprofloxacin from the investigated scaffold seemed to be in a sustained release profile with Korsmeyer-Peppas model as indicated by its higher $\mathrm{r}^{2}$-values (data not presented). Furthermore, the release of ciprofloxacin from the investigated scaffolds obeyed quasiFickian diffusion mechanism (n-values less than 0.5). This mechanism is based on hydrolysis as the polymer is hydrated, swell and then the drug diffuses through the swollen matrix system to the exterior, which ultimately slows down the kinetic release. It is also noted that by increasing drug content in the scaffold (5 to 10 and $20 \%$ ) the drug release was increased. This might be due to that higher drug content resulted in higher concentration difference between scaffold and the release medium which cause a higher drug release rate.

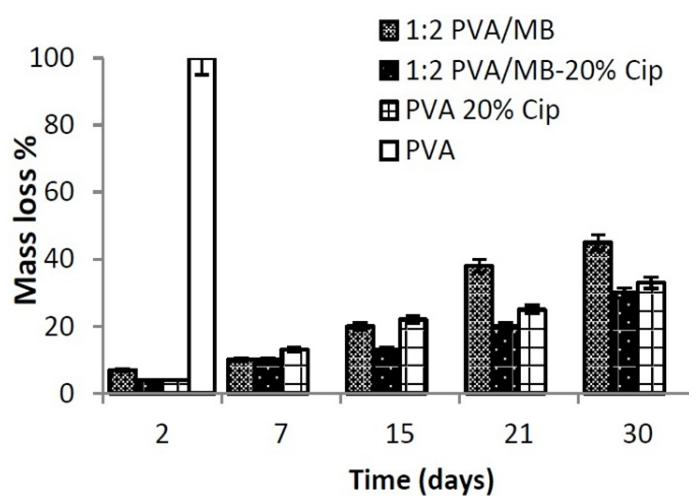

Figure 5: Biodegradation rate of the prepared scaffolds before and after drug loading. 
Citation: Mabrouk M, Mostafa AA, Oudadesse H, Mahmoud AA, Gaafar AM, et al. (2013) Fabrication, Characterization and Drug Release of Ciprofloxacin Loaded Porous Polyvinyl Alcohol/Bioactive Glass Scaffold for Controlled Drug Delivery. Bioceram Dev Appl S1: 009. doi: 10.4172/2090-5025.S1-009

Page 4 of 4

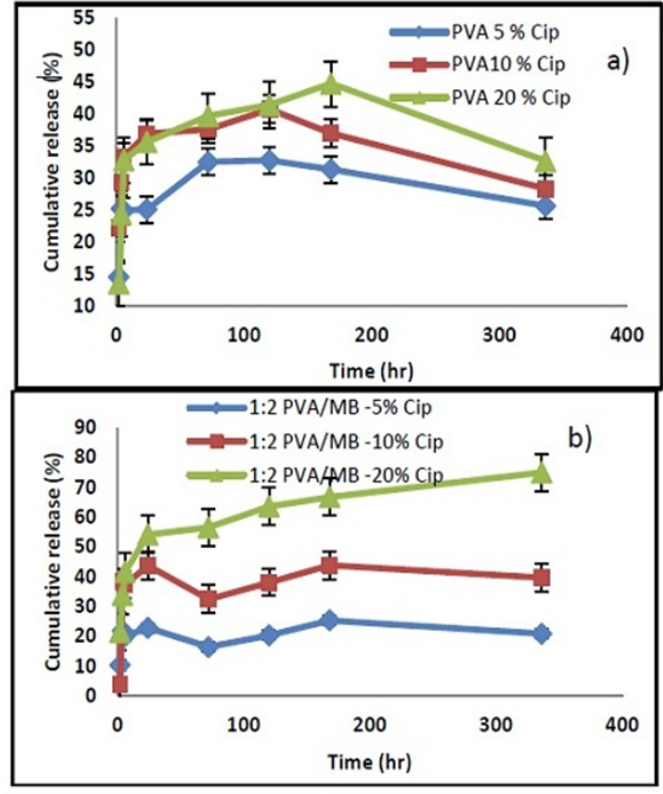

Figure 6: Ciprofloxacin release from [a) PVA and b) 1PVA:2MB] scaffolds.

Generally, incorporation of glass into scaffolds resulted in faster amount of drug released than its release from the PVA-based scaffold. A possible explanation for this observation is that the glass particles have occupied a huge part of the polymer matrix which leads to less compact structure causes higher and faster drug release [12,13].

\section{Conclusions}

Since polyvinyl alcohol (PVA) endows good fiber formation, biocompatibility, and chemical resistance properties, it can be used as a polymer additive to produce fibrous scaffold with BG.

In this study, the PVA/MB biocomposite scaffolds loaded with ciprofloxacin with well interconnected pore structure were fabricated via freeze drying technique. It is proved that addition of the drug added an advantageous of mechanical properties of the prepared scaffold with preserving the porosity through the induced cross linking without affecting of the drug efficiency.

The biodegradation rate and physicochemical properties of the prepared scaffolds could be controlled by controlling the glass content and the drug concentration added. Drug loaded scaffolds with ciprofloxacin exhibit a good drug delivery system with sustained drug release pattern.

\section{Acknowledgment}

This work was financially supported by Campus France and Academy of Scientific Research (ASRT) of Egypt, Imhotep program.

\section{References}

1. Yazdanpanah A, Reza K, Moztarzadeh A, Mozafari M (2012) Enhancement of fracture toughness in bioactive glass-based nanocomposites with nanocrystalline forsterite as advanced biomaterials for bone tissue engineering applications. Ceram Inter 38:5007-5014

2. Sokolsky-Papkov M, Agashi K, Olaye A, Shakesheff K, Domb AJ (2007) Polymer carriers for drug delivery in tissue engineering. Adv Drug Deliv Rev 59: 187-206.

3. Nayak AK, Sen KK (2009) Hydroxyapatite-ciprofloxacin minipellets for boneimplant delivery: Preparation, characterization, in-vitro drug adsorption and dissolution studies. Int J Drug Dev Res 1: 47-59.

4. Dietrich E, Oudadesse H, Lucas-Girot A, Mami M (2009) In vitro bioactivity of melt-derived glass $46 \mathrm{~S} 6$ doped with magnesium. J Biomed Mater Res A 88 1087-1096.

5. Yazdanpanah A, Reza K, Moztarzadeh F (2012) Enhancement of the fracture toughness in bioactive glass-based nanocomposites with nanocrystalline forsterite as advanced biomaterials for bone tissue engineering applications. Ceram Inter 38:5007-5014

6. Wong SC, Baji A, Gent AN (2008) Effect of specimen thickness on fracture toughness and adhesive properties of hydroxyapatite-filled polycaprolactone. Compos: Part A 39: 579-587.

7. Misra SK, Nazhat SN, Valappil SP, Moshrefi-Torbati M, Wood RJ, et al. (2007) Fabrication and characterization of biodegradable poly(3-hydroxybutyrate) composite containing bioglass. Biomacromolecules 8: 2112-2119.

8. Unnithan AR, Barakat NA, Pichiah PB, Gnanasekaran G, Nirmala R, et al. (2012) Wound-dressing materials with antibacterial activity from electrospun polyurethane-dextran nanofiber mats containing ciprofloxacin $\mathrm{HCl}$. Carbohydr Polym 90: 1786-1793.

9. Flautre B, Descamps M, Delecourt C, Blary MC, Hardouin P (2001) Porous HA ceramic for bone replacement: role of the pores and interconnections experimental study in the rabbit. J Mater Sci Mater Med 12: 679-682.

10. Rodri'guez-Tenreiro C, Alvarez-Lorenzo C, Concheiro A, Torres-Labandeira AA (2004) Characterization of cyclodextrincarbopol interactions by DSC and FTIR. J Therm Anal Calorim 77:403-411.

11. Zhang F, He C, Cao L, Feng W, Wang H, et al. (2011) Fabrication of gelatinhyaluronic acid hybrid scaffolds with tunable porous structures for soft tissue engineering. Int J Biol Macromol 48: 474-481.

12. Puga AM, Rey-Rico A, Magariños B, Alvarez-Lorenzo C, Concheiro A (2012) Hot melt poly- $\varepsilon$-caprolactone/poloxamine implantable matrices for sustained delivery of ciprofloxacin. Acta Biomater 8: 1507-1518.

13. Sunitha S, Kumar S (2010) Study on the effect of polymers on the release rate of drug from ciprofloxacin hydrochloride microspheres. J Pharmaceut Cosmetolol 1: 1-8.
This article was originally published in a special issue, International Symposium on Apatite and Correlative Biomaterials handled by Editor(s). Dr. Guy Daculsi, ISCM General secretary, France. 